\title{
Un dilatómetro para medir las variaciones de volumen del hormigón bajo carga
}

\author{
D. C. SPOONER

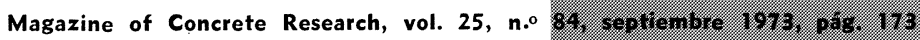 \\ (Traducción: SANCHEZ CASTRO, C. Titulado Téenico Especialliado I.E.T.e.c.)
}

Se describe un nuevo aparato que permite observar continuamente las variaciones volumétricas de la pasta de cemento o del hormigón bajo carga. Se fundamenta en el desplazamiento del agua desde un fuelle, que contiene una probeta sometida a la acción de la carga, hacia otro fuelle libre de acción alguna. La diferencia entre las medidas de los movimientos de los fuelles es proporcional a la formación volumétrica. Los resultados observados indican que existe una buena concordancia general con otras mediciones de la deformación volumétrica. Este aparato presenta algunas ventajas sobre otros métodos corrientes.

\section{N T R O D U C C C I O N}

Un material elástico, con un coeficiente de Poisson del orden de 0 a 0,5 , disminuye de volumen cuando se le somete a compresión. Aunque no se les puede considerar estrictamente elásticos, la pasta de cemento y el hormigón, cuyos coeficientes de Poisson son del orden 0,25 y 0,15 , respectivamente, también disminuyen de volumen cuando se someten a compresión uniaxil. Sin embargo en el caso del hormigón se ha podido observar que, en vez de seguir decreciendo de volumen con la aplicación creciente de tensión uniaxil, se alcanza un estado en el que el volumen empieza a aumentar. Esta dilatación prosigue con el aumento de la tensión hasta que se alcanza la tensión última (1). El comportamiento de las pastas de cemento es significativamente diferente a la del hormigón, puesto que bajo tensiones uniaxiles crecientes el volumen de una probeta de pasta de cemento sigue decreciendo al menos hasta que se alcanza la tensión máxima $(2,3)$. Esta diferencia de comportamiento entre el hormigón y la pasta de cemento se atribuye a la influencia del árido, llegándose a la conclusión de que el aumento de volumen del hormigón es causado por la propagación de las microfisuras $(2,4,5)$.

Continúa existiendo un considerable interés en utilizar las medidas de deformación volumétrica en la investigación de la fractura del hormigón, y, se ha diseñado y construido un nuevo dispositivo que presenta ciertas ventajas respecto a los métodos usuales para determinar la deformación volumétrica. Los métodos usuales para evaluar las deformaciones volumétricas que se producen cuando se somete a carga a las probetas de hormigón o de pasta de cemento consisten en medir las deformaciones de la superficie axil y laterales y relacionar éstas con la deformación volumétrica por aproximación.

Deformación volumétrica $=$ Deformación axil +2 (deformación lateral).

(La contracción se considera aquí como positiva y así en la compresión uniaxil la deformación axil es positiva y la deformación lateral es negativa). 
Las deformaciones superficiales laterales y axiles normalmente se miden por medio de transductores o "straing gauges" de resistencia eléctrica. Las desventajas de este método son que:

1) sólo una pequeña zona de la probeta sirve de testigo para proporcionar una medida de la deformación volumétrica y así, especialmente al aproximarse a la tensión última, las variaciones en la deformación de la probeta pueden dar lugar a inexactitudes en la determinación de la deformación volumétrica;

2) no es posible medir las deformaciones volumétricas con veracidad por estos métodos en la proporción de rama descendente de la curva tensión-deformación, debido a agrietamiento superficial;

3) cuando se utilizan gauges de resistencia es necesario fijar nuevos gauges a cada probeta antes del ensayo, y éstos no pueden utilizarse nuevamente.

El nuevo aparato, que se describe más adelante, mide el cambio de volumen total de la totalidad de una muestra de hormigón o de pasta de cemento; puede ser utilizado en la proporción descendente de la curva tensión-deformación del hormigón.

\section{EL DILATOMETRO}

El dilatómetro es un perfeccionamiento del aparato descrito por Edmond y Paterson (6) para medir las deformaciones volumétricas de las rocas sometidas a alta presión cohibida. Se basa en el desplazamiento del agua desde un fuelle flexible de acero inoxidable que contiene a la probeta sometida a compresión, hasta otro fuelle idéntico que se puede mover libremente. $\mathrm{El}$ aparato se presenta en esquema en la figura 1 y en fotografía en la figura 2 .

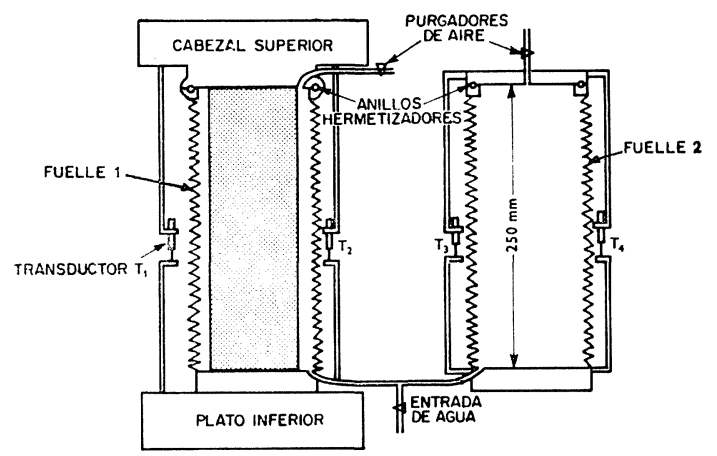

Fig. 1.-Vista esquemática del dilatómełro.

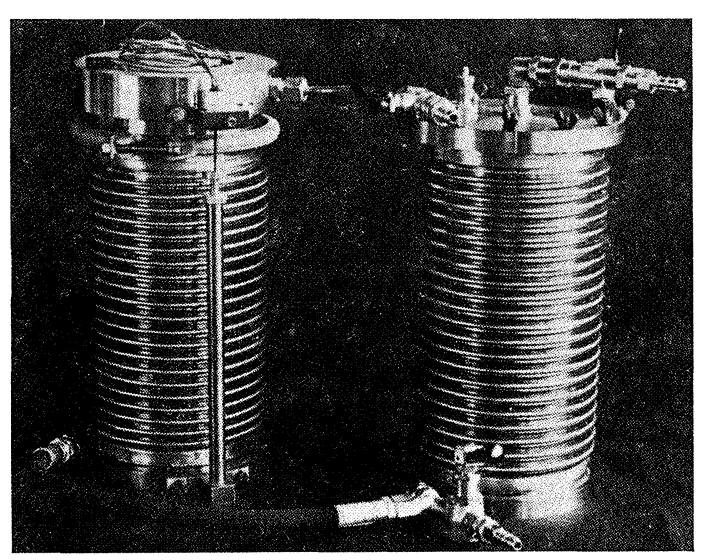

Fig. 2.-Conjunto del dilatómetro.

Si en el fuelle 1 no hay probeta, cualquier acortamiento del fuelle 1 se traduce en un alargamiento del fuelle 2. Por consiguiente, un desplazamiento axil de una probeta en el fuelle 1 viene acompañado por un desplazamiento equivalente del fuelle 2 , pero de signo opuesto. Además, cualquier variación volumétrica de la probeta da lugar a un desplazamiento axil adicional del fuelle 2, que bien puede aumentar o disminuir el desplazamiento total del fuelle 2, dependiendo esto del signo de la deformación volumétrica. En consecuencia, la diferencia entre los desplazamientos axiles de los dos fuelles es proporcional a los cambios de volumen de la probeta. La deformación volumétrica real de la probeta es fácilmente determinable si se conoce el área de la sección transversal de los fuelles. 
En cada uno de los fuelles los desplazamientos axiles se midieron utilizando dos transformadores diferenciales, montados en lados opuestos de los fuelles y con una sensibilidad lineal de 1,25-0-1,25 mm y un voltaje correspondiente de salida de $\pm 5 \mathrm{~V}$. (En la figura 2 puede verse un solo transductor). Las salidas de los dos transductores montados en cada fuelle se sumaron y las salidas totales de cada fuelle se conectaron en serie. La figura 3 muestra el circuito.

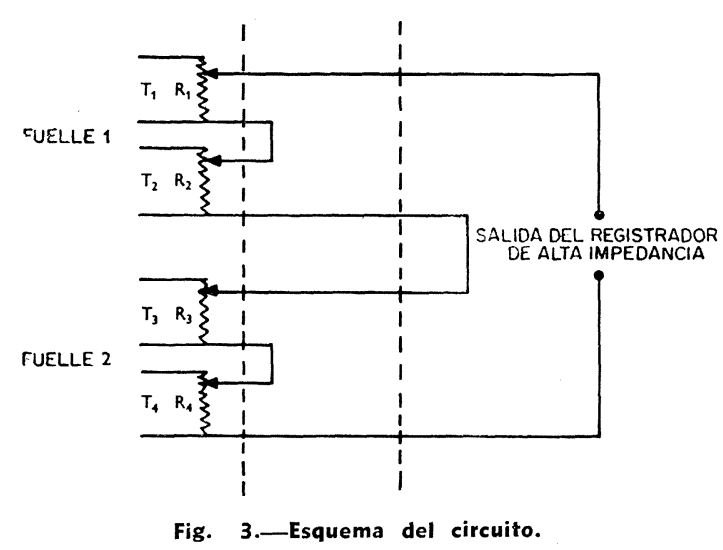

para dar una salida con voltaje cero, es decir, que hubiese ninguna probeta en el fuelle 1.

Las salidas totales de los fuelles 1 y 2 serán las mismas, pero de signo opuesto, en este caso, de modo que cuando son compuestos en el registrador no indicará ninguna señal en el registrador de desplazamientos axiles que normalmente son utilizados en el ensayo de una probeta. Cualquier pequeña y clara señal debida a variaciones dimensionales entre los fuelles se reducirá a cero mediante unos ligeros ajustes en $R_{1}$ y $R_{2}$ o en $R_{3}$ y $R_{4}$.

Antes de colocar la probeta seca en el fuelle 2, se selló contra la penetración de agua para lo cual se introdujo en una fina manga de caucho. Los extremos de la manga se pegaron a dos platillos de acero de la misma sección transversal que la probeta. No es necesario el sellado cuando las probetas están saturadas, aunque se han observado pequeñas diferencias cuando los resultados de las probetas saturadas y selladas se comparan con los resultados de las probetas saturadas sin sellar.

\section{UTILIZACION DEL DILATOMETRO}

El dilatómetro ha sido diseñado para acomodar probetas de $250 \times 75 \times 75 \mathrm{~mm}$ y en el ensayo que se describe se utilizó una prensa con servo-control con velocidad de deformación constante. La prensa no tendrá dispositivo de asiento de bola y la alineación de la probeta en la máquina se obtendrá utilizando una placa de asiento de resinas epoxi dispuesta entre la probeta y el plato superior de la prensa (3). Parece que no existe razón en contra para que el dilatómetro no funcione satisfactoriamente con otros procedimientos de control y con prensas provistas con asiento de bola en el plato.

El agua utilizada en el dilatómetro es agua limpia corriente, que se deja toda la noche reposar en el laboratorio con objeto de alcanzar la temperatura ambiente. Se tomaron precauciones similares con las probetas. Con objeto de hacer las capacidades térmicas de los dos fuelles lo más iguales posibles, se colocó en el fuelle 2 una probeta saturada, de hormigón, en lugar de sólo rellenarlo con agua. Esta probeta falsa no deberá tocar la parte superior del fuelle 2. Una vez que se han adoptado estas precauciones, se ha visto que 
la tendencia de la señal de salida que representan deformaciones volumétricas no constituyen un problema, y con adecuados dispositivos de registro puede conseguirse fácilmente una sensibilidad de $100 \times 10^{-6}$ deformación volumétrica por centímetro.

\section{DISCUSION DE LOS RESULTADOS PRELIMINARES}

En la figura 4 se dan los resultados típicos obtenidos con probetas de hormigón y pasta de cemento. Ambas curvas concuerdan muy bien con los resultados obtenidos para ambos tipos de probetas y, en consecuencia, tienden a confirmar la validez tanto del nuevo aparato como del método usual para determinar la deformación volumétrica. El resultado obtenido con la probeta de hormigón muestra el típico comportamiento a la dilatación después de un período inicial de consolidación. La dilatación continuó en tanto fue incrementándose la deformación axil, es decir, muy por encima de la tensión última que resistió la probeta. Una disparidad entre los resultados para el hormigón y otros datos publicados se refieren al cambio de tensión de la pendiente inicial de la relación volumen/tensióndeformación.

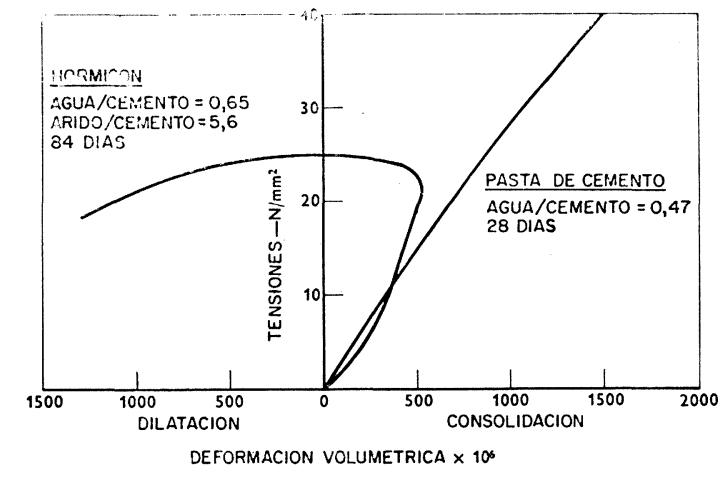

Fig. 4-Cambios volumétricos para las probetas de pasta de cemento y de hormigón. Resultados típicos.

En la figura 4 la porción inicial de la curva de probeta de hormigón es cóncava respecto al eje de las tensiones, es decir, la pendiente continúa incrementándose, mientras que la mayoría de otros datos para el hormigón muestra que esta parte es recta o convexa respecto al eje de las tensiones. La razón para esta diferencia de carácter no se conoce todavía, pero se estudiará más adelante, puesto que conduce a un replanteamiento del problema de la irrupción de las microfisuras en el hormigón.

La curva deformación volumétrica de las probetas de pasta de cemento indica una continua disminución en el volumen de la probeta hasta que se alcanza la tensión última. En este punto el sistema de servo-control es incapaz de impedir la rotura explosiva de la probeta.

\section{O N C L U S I O N E S}

Los ensayos de prueba con un dilatómetro que mide directamente la deformación volumétrica producida en la pasta de cemento o en el hormigón bajo cargas, muestran que se pueden comparar bien con otros resultados ya publicados. A diferencia con otros métodos de medir la deformación volumétrica; éste es capaz de dar resultados fidedignos, incluso cuando la superficie de la probeta esté fisurada, puesto que, por ejemplo, puede hacerlo en la parte más alta de la curva tensión-deformación a compresión. 


\section{AGRADECIMIENTO}

El autor agradece plenamente el trabajo de Mr. A. E. Read por el diseño y construcción del dilatómetro.

Este trabajo forma parte de un curso de la Universidad de Londres dirigido para el grado de Doctor en Físicas bajo la supervisión de Mr. C. D. Pomeroy, de la Asociación del Cemento y del Hormigón, y del Dr. J. W. Dougill, del King's College, de Londres.

\section{R E F E R E N C I A S}

(1) Reinius, E.: Una teoría sobre la deformación y rotura del hormigón. Betong. Vol. 40, n. 1 , 1955, pp. 15-43, Londres. Cement and Concrete Association. Library Translation. Cj. 63.

(2) ShaH, S. P. y Chandra, S.: Tensión crítica, variación de volumen y mocrofisuración del hormigón Journal of the American Concrete Institute. Proceedings Vol. 65, n. ${ }^{\circ}$ 9, septiembre 1968, pp. 770-781.

(3) Spooner, D. C.: La relación tensión-deformación en las pastas endurecidas de cemento sometidas a compresión. Magazine of Concrete Research. Vol. 24. n. ${ }^{\circ}$ 79, junio 1972, pp. 85-92.

(4) Hsu, T. T. C., Slate, F. O., Sturman, G. M. y Winter, G.: La microfisuración del hormigón en masa y la forma de la curva tensión deformación. Journal of the American Concrete Institute. Proceedings, Vol. 60, n. ${ }^{\circ}$, febrero 1963 , pp. 209-224.

(5) Newman, K y Newman, J. B.: Teoría de la rotura y criterio de diseño para el hormigón en masa. Structure, solid mechanics and engineering design: Proceedings of the Southampton 1969. Civil Engineering Materials Conference. Editor: M. Te'eni. London, Wiley-Interscience, 1971. Vol. 2. Paper 77, pp. 963-995.

(6) Edmond, J. M. y Paterson, M. S.: Cambios de volumen durante la deformación de las rocas sometidas a altas presiones. International Journal of Rocck Mechanics and Mining Sciencies. Vol. 9, n. ${ }^{\circ}$, marzo 1972, pp. 161-182. 